\title{
Extended time improves reading comprehension test scores for adolescents with ADHD
}

\author{
Thomas Edwards Brown ${ }^{*}$, Philipp Christian Reichel, Donald Michael Quinlan
}

Department of Psychiatry, Yale University School of Medicine, New Haven, USA.

Email: * thomas.e.brown@yale.edu

Received 29 August 2011; revised 20 September 2011; accepted 15 October 2011.

\section{ABSTRACT}

Objective: To test the hypotheses that reading comprehension difficulties of adolescent students with ADHD: 1) are related not so much to weak verbal abilities or weak basic reading skills, as to impairments of working memory and processing speed characteristic of ADHD; and 2) that extended time on a test of reading comprehension would yield significantly higher reading comprehension scores than would standard time. Method: Charts of 145 adolescents 13 - 18 years diagnosed with DSM-IV ADHD and no specific reading disorder after a comprehensive clinical and psycho-educational evaluation, were reviewed to extract 1) word reading and word attack subtest scores from the Woodcock-Johnson Achievement Test or the Wechsler Individual Achievement Test; 2) Index scores from WISC-IV or WAIS-III IQ tests; 3) scores from the Nelson-Denny Reading Test. Results: Mean index scores for verbal comprehension abilities not including reading were in the high average range, but working memory and processing speed index scores were significantly weaker. Under standard time limits $\mathbf{5 3 \%}$ were unable to complete the reading comprehension test and only $42.8 \%$ were able to score within 1 SD of their IQ verbal comprehension index (VCI). When allowed extended time, $77.9 \%$ were able to score within 1 SD of their VCI. T-test comparisons between standard time and extended time were significant at $<\mathbf{0 . 0 0 1}$. Conclusions: Allowing extended time for adolescents with ADHD to complete tests involving reading may help to compensate for their impairments of working memory and processing speed, allowing them to score closer to their actual verbal abilities.

Keywords: ADHD; Reading Comprehension; Extended Time; Working Memory; Processing Speed

\section{INTRODUCTION}

Among adolescents with ADHD are some who report chronic difficulties in reading that significantly impair their ability to complete tests and assignments within usual time allotments. Although most demonstrate no significant impairment in phonological processing, the usual hallmark of dyslexia, these students complain of chronic slowness in assigned reading, due usually to a need to re-read passages several times in order fully to grasp the meaning. They also report that, though they may understand the content at the time of reading a passage, they have chronic difficulty in recalling what they have read just a few minutes earlier. It appears that re-reading is needed to engage their focus sufficiently to encode the information in memory. One student with ADHD described this: "Most of the time when I'm reading assignments in my textbooks, I'm just licking the words rather than chewing them. That's why I have to keep going back to read it all over again."

Interestingly, many of these students report that such impairments often are not present when reading selfchosen rather than assigned texts. This clinical observation suggests that such reading impairments may be the result of impairments in executive functions (EF), which tend to be situationally specific, rather than consistent impairment in verbal abilities or basic reading skills. Pennington [1] and Brown [2,3] have described the situational variability of executive functions impaired in ADHD, how individuals with ADHD often demonstrate little impairment in their ability to deploy executive functions when doing tasks which hold strong personal interest or anxiety for them, though they show much EF impairment in most other situations. This is consistent with findings by Anmarkrud and Braten [4] that students' motivation for reading content of personal interest to them, the value they place on reading a specific text, plays an important role in their reading comprehension.

In this study, we hypothesized that adolescents with 
ADHD who are slow in reading comprehension tasks and do not have a specific learning disability in reading would demonstrate relative weaknesses in working memory and processing speed, aspects of executive function often impaired in ADHD. Further, we hypothesized that extended time on a test of reading vocabulary and reading comprehension would help these students to compensate for their ADHD-related reading impairments, yielding higher reading comprehension scores more consistent with the individual's verbal comprehension abilities as shown on an IQ measure not involving reading.

There is considerable evidence that executive functions often impaired in ADHD, especially processing speed and working memory, play an important role in reading, particularly in reading fluency and comprehension (see Willcutt [5], Shanahan [6], Laasonen [7], McGrath [8], Arnell [9] and Swanson [10]). This is true not only in those with a reading disorder, but also in those who are not impaired in phonological processing (see: Sesma [11], Locasio [12], Samuelson [13], Jacobson [14], Bental [15] and Leong [16]).

One specific executive function important in reading comprehension is processing speed. Willcutt et al. [17] demonstrated that impairment in processing speed is found much more in children with reading disability (dyslexia), in children with ADHD, and in those with both disorders than in controls. In a sample of children and adolescents Shanahan et al. [6] demonstrated that processing speed, measured in multiple ways, is a shared cognitive risk factor across reading disorder and ADHD with a correlation of 0.7 between the two disorders. The authors suggested that participants with each disorder may be slowed down because they are engaging in a speed-accuracy trade-off, buying increased accuracy with a slower rate.

The study by Shanahan et al. utilized a variety of measures to assess processing speed. These included linguistic measures such as rapid automatized naming (RAN) and the Stroop test as well as non-linguistic measures such as the WISC Coding subtest, the Trailmaking test and the Stop-Signal task. Findings indicated that, despite their differences, these various measures of processing speed were highly correlated with one another, all apparently reflecting a common factor that contributes to both ADHD and Reading Disorder.

Similar findings in a sample of adults in Finland were reported by Laasonen, Leppamaki, Tani and Hokkanen [7]. Their assessments of adults with dyslexia, attention deficit disorder, and comorbid ADHD with dyslexia found that all shared relative weakness in processing speed as measured by the WAIS-III.

Primary importance of processing speed in overlap between ADHD and reading disorder was also demons- trated in a study by McGrath et al. [8]. They used multiple deficit modeling with a sample of children with ADHD and/or Reading Disorder. Their analysis identified processing speed as the most important common factor between inattention and reading.

One measure of processing speed is the rapid automatized naming (RAN) test, a timed measure of speed and accuracy for naming familiar stimuli, e.g letters, digits, colors, etc. randomly sequenced. The RAN has been shown in numerous studies to predict reading comprehension. Arnell and colleagues [9] recently reported a study using a sample of university undergraduates that identified specific cognitive elements of the RAN that contribute to its shared variance with reading comprehension. Their various measures explained $52 \%$ of the variance shared by the RAN and reading comprehension as measured by the Nelson-Denney Reading Test (NDRT). Results suggested that working memory encoding is a significant component of the relationship between processing speed measured by the RAN and reading ability.

Both processing speed and working memory have been identified as important aspects of the complex cognitive processes involved in reading comprehension. Cain and Oakhill [18] reviewed multiple studies which demonstrate that for skilled readers as well as for those with poor reading skills or very limited reading comprehension, working memory plays a critical role in integrating information to facilitate comprehension of text. This is likely to be because comprehension depends upon recalling what has been read in preceding sentences and paragraphs so that the reader can develop and modify an adequate working understanding of the message of each section of the text and of how those components are related to one another.

Swanson, Zheng and Jerman [10] published a metaanalysis of studies on working memory (WM), shortterm memory (STM) and reading disabilities. They defined WM as "a processing resource of limited capacity involved in the preservation of information while processing the same or other information (p. 260)"; they differentiated this from STM, a resource in which small amounts of information are held passively and then produced in an untransformed fashion, like the buffer of a printer holding information from a computer prior to printing. They noted that WM measures in these studies were related primarily to reading comprehension of sentences and paragraphs while STM measures related primarily to recognition of individual words on a list.

Sesma, Mahone and colleagues [11] extended this view in a 2009 study, noting that while some children have difficulty with reading comprehension because they have chronic difficulty in decoding and accurately reading single words, there are others who struggle with reading 
comprehension despite their having adequate ability to decode and accurately read single words. These researchers did a study with 60 children aged 9 to 15 years with deficits in reading comprehension to test the hypothesis that executive functions would be significantly associated with reading comprehension skills, but not with single word reading accuracy in children with word reading deficits, reading comprehension deficits, and/or ADHD.

Results indicated that executive skills differentially support reading comprehension, but are less necessary for single word reading. They explained their findings as follows: "Reading comprehension is inherently more complex than single word reading, with demands that go beyond phonological decoding and word identification and include higher order cognitive processing of meaning conveyed through sentences and paragraphs... executive control skills such as planning and working memory become more necessary as the length and complexity of written text increases" (p. 8). A subsequent study by these authors [12] of executive function impairments in children with reading comprehension deficits provided additional evidence that executive function impairments, including planning and organizing, were closely associated with impairments in reading comprehension. A significant proportion of the samples in both of these studies were diagnosed with ADHD.

Similar findings about the role of executive function impairments in reading comprehension of individuals with ADHD was reported by Samuelsson, Lundberg and Herkner [13]. In their study of male adults they found a significant correlation between poor reading comprehendsion and ADHD while there was no significant association between word decoding and ADHD. They explained this by arguing that word decoding "is determined by a smoothly operating, encapsulated... phonological module largely unrelated to higher cognitive functions such as executive controls" while "reading comprehension involves many of the higher cognitive control functions assumed to be impaired in ADHD" (p. 165). In a sample of 9 to 14 year old children with ADHD Jacobson, et al. [14] (2011) demonstrated that both processing speed and working memory were significantly associated with reading fluency.

The importance of working memory and processing speed in reading comprehension is not limited to reading in the English language. Using Hebrew language measures, Bental and Tirosh [15] evaluated a sample of Israeli boys with ADHD only, Reading Disorder only, comorbid ADHD + Reading Disorder, and controls, all of whom were equivalent in oral language functions. They demonstrated that reading performance in Hebrew by preadolescent children with ADHD is linked to rapid nam- ing and to executive functions, particularly verbal working memory, more than to phonological processing. A large sample of preadolescent Chinese children showed a similar pattern in a study by Leong, et al. [16] who found that verbal working memory had a strong unique effect on Chinese text comprehension, significantly greater than the influence of their ability to read pseudowords or rapid automatized naming (RAN).

Increasing recognition of the importance of working memory and processing speed in reading comprehension is consistent with a major shift emerging from research on dyslexia. Shaywitz and Shaywitz [19] have emphasized how current research shows that reading is not simply a modular process dependent only on phonological processing needed to decode words. They argued that reading must now be understood as involving also attentional mechanisms that are essential to fluency and automaticity in reading. "The critical requirement for automaticity is for the reader to encode the relevant items in memory and to retrieve them on a subsequent encounter... for both encoding and retrieval, attention is central (p. 1332)."

The Shaywitz paper also notes the importance of higher association cortices, particularly the prefrontal cortex, in attentional mechanisms. Recognizing that “... attentional mechanisms play a critical role in reading and that disruption of attentional mechanisms plays a causal role in reading difficulties (p. 1343)", Shaywitz and Shaywitz suggested that medications shown effective for improveing attentional function in patients with ADHD “... might be an effective adjunct to improving reading in dyslexic students (p. 1329)." They also noted that "... lack of automatic, fluent reading means that the dyslexic reader may be able to decode words, but is still not able to read quickly and continues to be at a disadvantage compared to non-dyslexic peers when taking high-stakes standardized tests such as SATs, Graduate Management Admission Tests, Graduate Record Examination and so forth (p. 1343)."

The comments of Shaywitz and Shaywitz focus on dyslexic readers, but they are applicable to non-dyslexic readers as well. Recent research such as studies cited earlier in this paper clearly indicates that adequate reading comprehension depends not only on ability to recognize and decode words. It also depends upon 1) adequate attention; 2) adequate working memory; and 3) adequate processing speed. Typically, individuals with ADHD are significantly impaired in all 3 of these critical executive functions. One example is a study of fluency and reading comprehension by Jacobson and colleagues [14] which demonstrated that slow processing speed and impaired working memory were significant predictors of difficultties in reading fluency in children aged 9 to 14 years 
with ADHD.

Our study reported here focused not on students who suffer from dyslexia, but a sample of adolescents aged 13 to 18 years who did not suffer from a reading disorder. This is an older group than those in most studies of reading comprehension, an age group whose academic work usually requires reading of longer, more complex texts. All had been carefully diagnosed with ADHD and were without a comorbid reading disorder. Students with scores for basic reading skills below the low average range were excluded from the study. We tested several predictions about cognitive functions related to reading comprehension difficulties in these adolescent students diagnosed with ADHD. We also tested the effects of one specific compensatory strategy that may be helpful to many of these students... extended time for a reading comprehension test.

Walczyk and colleagues [20] have reported research testing a variety of compensatory strategies that have been demonstrated helpful for readers at various skill levels who are struggling for efficient comprehension of a text. These compensatory strategies include slowing down the reading rate; pausing to allow more time for processing; looking back in the text to clarify confusion; jumping over text segments that are confusing, but not essential; and rereading of the text to enhance understanding. Regression analyses in their study revealed that restriction of time to read a text tends to reduce comprehension because it does not allow sufficient opportunity for the reader to clarify information to be processed in working memory. This is consistent with clinical reports of many students with ADHD who are unable to complete exams within standard time allocation. Extended time is one useful way to help students whose reading comprehension is compromised by impaired working memory and processing speed.

We predicted that students diagnosed with ADHD would tend to be significantly weaker in working memory and processing speed than in their overall verbal abilities exclusive of reading. This prediction was consistent with findings of our study on executive function impairments in children and adolescents with ADHD. It was also consistent with findings reported by Mayes and Calhoun [21] in their report of IQ index score predictors of academic achievement. Their study used index scores from WISC-III/IV to predict learning disorders (LD) with a discrepancy formula using the Wechsler Individual Achievement Test-Second Edition (WIAT-II). From a large sample of youths 6 - 16 yrs they reported that the most powerful predictor of academic achievement was the Verbal Comprehension Index and that the most powerful predictors of LD were the index score for Working Memory/Freedom from Distractibility and the index score for Processing Speed.
We also predicted that our sample of students with ADHD and without a specific LD in reading would have relatively unimpaired basic reading skills (within $1 \mathrm{SD}$ of their verbal comprehension index on the WISC/ WAIS) but would tend to score relatively low on a timed test of reading comprehension $(\geq 1$ SD below their verbal comprehension index). When allowed extended time on a reading comprehension test, we predicted that their score would be closer to their VCI.

\section{METHODS}

\subsection{Sample}

Records of two ADHD specialty clinics in a metropolistan area of the Northeast U.S., one private, the other in a university medical center were reviewed to select charts of all individuals 13 to 18 years who sought consultation for attention or learning problems, met DSM-IV diagnostic criteria for ADHD, had undergone a full psycho-educational evaluation within the past three years, and did not have any specific learning disorder in reading.

Potential participants were excluded if their basic reading skills as measured by WIAT-II or WJ-III word reading or pseudoword decoding (word attack) scores were below 80 , or if their vcrbal comprehension index score on the WISC or WAIS was below 80 . Thus participants were all non-dyslexic students with verbal comprehension abilities (not including reading) in the low average range to superior range who suffered significant current impairments from ADHD sufficient to warrant diagnosis under DSM-IV diagnostic criteria. The sample included 145 participants aged 13 to 18 years; $69.1 \%$ were males. The mean for VCI was 118.6 ; the mean for POI was 112.6.

Evaluation protocols in both clinics were identical. A licensed clinical psychologist experienced in assessing ADHD and related disorders conducted a two hour clinical interview of the patient with one or both parents to take relevant history and assess impairment according to DSM-IV-TR diagnostic criteria for ADHD. A normed and validated rating scale for ADD and related executive functions, the Brown ADD Rating Scale, [22,23] was administered and screening for possible comorbid disorders was completed. In a separate session after ADHD diagnosis, the full WISC-IV [24] or WAIS-III [25] IQ tests were administered followed by the Wechsler Individual Achievement Test (WIAT-II) [26] or the Woodcock-Johnson 3rd Edition Achievement Tests [27].

\subsection{Measures}

To assess basic reading skills, we used standardized measures of word reading and pseudoword reading (word attack) from the Woodcock-Johnson Achievement Test 
(WJ-III) or the Wechsler Individual Achievement Test (WIAT-II). These were compared with the student's overall verbal abilities as measured by the Verbal Comprehension Index (VCI) of the student's WISC or WAIS IQ tests, a measure of verbal ability not requiring reading. The VCI was used as a basis for comparison because it is a validated and normed measure of verbal comprehension abilities that does not require any reading.

To assess processing speed and working memory, we used two index scores from the student's WISC or WAIS: Working Memory Index (WMI) and Processing Speed Index (PSI), neither of which involves reading, Mayes and colleagues [28] have shown, in their own research and have cited other studies showing that children with ADHD tend to score significantly lower on WMI and PSI than comparison children and significantly lower than their own Verbal Comprehension index.

To assess the impact of extended time for reading comprehension, we used the Nelson-Denny Reading Test [NDRT] [29], a normed measure which requires each subject to complete 80 multiple choice vocabulary questions within $15 \mathrm{~min}$. Each is then asked to read 7 narrative passages and answer 38 multiple choice comprehendsion questions within $20 \mathrm{~min}$. Any individual unable to complete the vocabulary section is allowed up to 9 additional minutes; anyone unable to complete the comprehension section is allowed up to 12 additional minutes.

\subsection{Data Analysis}

To allow for comparison of NDRT and WAIS/WISC scores, the percentile ranks of the NDRT were converted to a normal-curve scale that matched the mean (100) and SD (15) of IQ and Index scores. We scored results obtained with extended time using the NDRT norms for standard time. Our procedure differs from the Extended Time tables used in the NDRT, but our procedure with this scoring is similar to that of Ofiesh, et al. [30] and closely mirrors what is done when the accommodation of extended time is allowed, i.e., scores are ranked on the same metric. Students given extended time accommodations for standardized measures such as the SAT and ACT are scored on the same metric as students without accommodations. They are not penalized by being scored with separate norms applicable only to those receiving extended time.

We compared each student's scores for reading vocabulary and reading comprehension on the NDRT under both standard time and extended time conditions (using the NDRT norms for standard time) with their basic reading abilities and their overall cognitive abilities as measured by the VCI. We then used paired t-tests to assess significance.
We chose the verbal comprehension index as the standard for comparison because it is an age-based and validated measure of verbal comprehension abilities that does not require any reading. Normative data from the WIAT-II show that VCI is a reasonable predictor of reading comprehension. Only $11 \%-12 \%$ of individuals score more than 1 standard deviation below their WISC/WAIS VCI on a standardized measure of reading comprehension [26].

\section{RESULTS}

Overall verbal skills of these students on tests not involving reading were relatively strong.

As shown in Figure 1, their WISC-IV/ WAIS-III Verbal Comprehension Index (VCI) mean was in the high average range: 118.6 (13.8). As predicted, and as is common among individuals diagnosed with ADHD, their mean index scores for Working Memory (WMI) and Processing Speed (PSI) were both more than 1 SD lower, in the average range: WMI: 102.8 (14.2); PSI: 99.9 (16.3). These scores indicate relative weakness in these two index scores that tap executive functions associated with ADHD and are significantly lower than the VCI ( $p$ $=<0.0001)$.

It should be noted that this sample was not strong only in verbal comprehension and weak in all other factors of measured IQ. Their mean score for Perceptual Organization Index (POI) was in the high average range, 112.6 (12.6), just 6 points lower than their mean VCI. This discrepancy is not significantly different from the standardization samples for the WISC-IV or WAIS-III [26]. These students were in the high average range for the two factors of Wechsler IQ tests less sensitive to im- pairments of executive function. Like most individuals with ADHD, they were relatively weak in their scores for WMI and PSI, the two factors of these IQ tests that are more sensitive to executive function impairments [21].

Also shown in Figure $\mathbf{1}$ are mean scores for basic

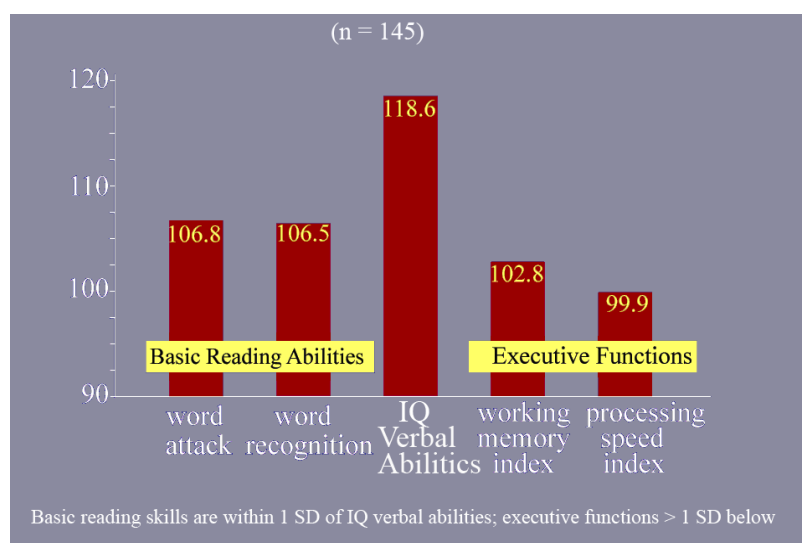

Figure 1. Basic reading vs. IQ verbal abilities vs. executive functions. 
reading skills on the WJ-III/WIAT-II, word recognition and word attack, which were solidly in the average range: word recognition: 106.5 (11.05) word attack: 106.8 (13.4). These scores indicate that basic reading skills of this sample were somewhat lower than their VCI, but still solidly in the average range. Reading difficulties of this group were not due to significant impairments in ability to use phonics principles to decode unfamiliar words or to impairments in ability to recognize and correctly pronounce words from an age-appropriate vocabulary list.

This is consistent with the findings of other studies (see: Sesma [11], Locasio [12] and Jacobson [14]) demonstrating that problems with reading comprehension exist independently in some students with fully adequate basic reading skills. Reading comprehension problems are not limited to those with weak decoding and/or sight vocabulary. Reading comprehension requires adequate decoding skills, but it also requires adequate executive functioning, especially in working memory and processing speed, two areas typically weak in individuals with ADHD.

Figure 2 displays comparisons of participants' mean scores for VCI and their mean scores for the NDRT under standard and extended time. Scores for the vocabulary section with standard time were 10.6 (15.1) points below their VCI. When those who were unable to finish the vocabulary section were allowed extended time, the discrepancy between mean VCI and mean vocabulary score reduced to 8.2 points (12.1).

Under standard time the mean score for the NDRT comprehension section was 17.7 points lower than the mean VCI. As predicted, scores of these ADHD students improved significantly when they were allowed the extended time on the reading comprehension section, yielding a discrepancy of just 7.4 (13.1). Paired t-tests on both comparisons yielded $\mathrm{p}=<0.0001$.

In addition to comparing group means, we also assessed individual performances on these measures. Each

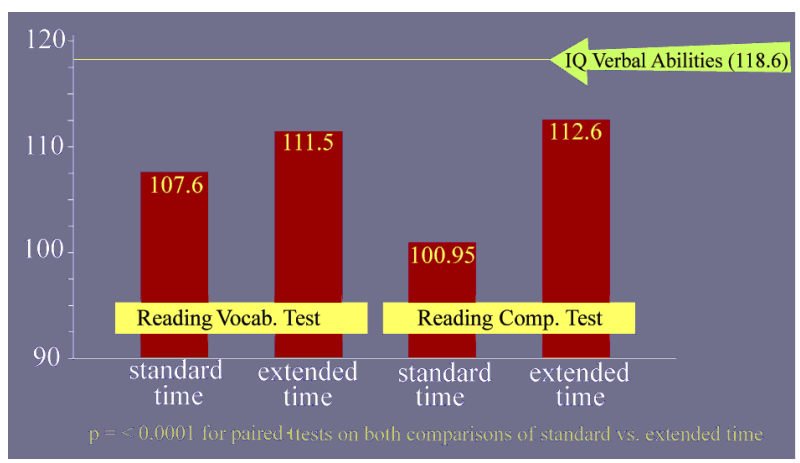

Figure 2. Standard vs. extended time vs. IQ verbal abilities group means. participant's score for vocabulary and for comprehension on the NDRT under standard time was compared with his scores on these measures when allowed extended time. In both cases, obtained scores on the NDRT were compared with that individual's VCI score on the IQ test. Thus we assessed the degree to which that individual's performance on the NDRT resembled his score for basic verbal comprehension abilities.

Figure 3 shows that under standard time conditions for vocabulary items, $63.4 \%$ of the sample obtained a score on the NDRT within one SD of their VCI score; for reading comprehension items, only $42.8 \%$ were within that range. This can be compared to the normative data from the WIAT which shows that only $11 \%-12 \%$ of individuals scored one SD or more below their VCI on a standardized measure of reading comprehension.

When those unable to finish in standard time allotments were allowed the stipulated amount of extended time to finish, scores within 1 SD of their VCI were obtained by $72.9 \%$ for vocabulary and by $77.9 \%$ for comprehension, much closer to the percentage in the general population that would be expected to have such a discrepancy.

\section{DISCUSSION}

While many of these 145 adolescents with ADHD were able to complete the NDRT within standard time constraints, $48 \%$ were unable even to attempt all the vocabulary questions and $53 \%$ were unable to attempt all of the reading comprehension questions without extended time. This is consistent with our clinical experience that many, but not all students with ADHD report chronic difficulty in completing tests, particularly tests involving substantial reading, within standard time limits.

The high average mean verbal comprehension index scores of this sample and their solidly average mean scores for basic reading skills may limit generalizability of our results, but they do support our prediction that

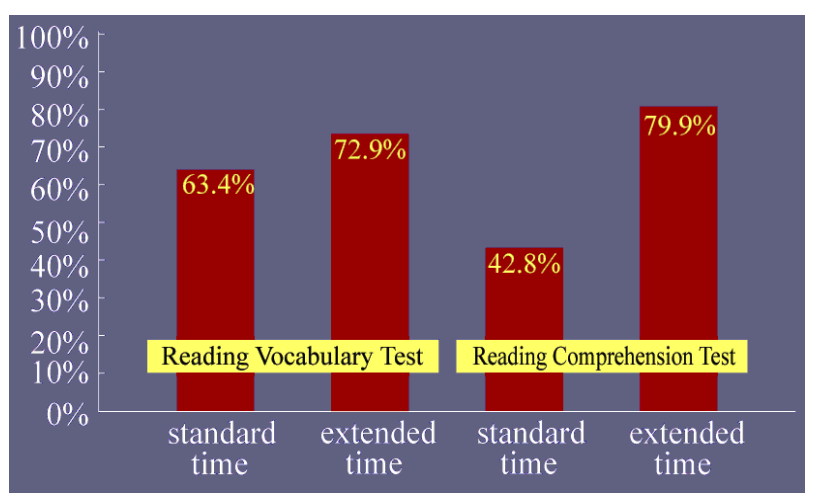

Figure 3. Percentage of subjects scoring within $1 \mathrm{sd}$ of their iq verbal ability index standard time vs. extended time. 
their difficulty with the reading test used was not likely to be a result of weaknesses in basic verbal abilities or weaknesses in basic reading skills. It appears more likely that their significant relative weaknesses in processing speed and working memory, aspects of the executive functions often impaired in ADHD, contributed to their relatively low scores on our reading measure administered with just standard time.

These data provide evidence that on tests involving reading longer, more complex texts under time constraints, allowing a modest extension of time can provide individuals with ADHD opportunity to demonstrate reading comprehension abilities more consistent with their actual verbal abilities. This accommodation can help these students to compensate for the chronic problems with working memory and processing speed that, for such tasks, burden many, though not all, adolescents with ADHD.

Some might question whether allowing extended time on exams, particularly high stakes exams that may impact admission to university or graduate studies, would be providing ADHD students with an unfair advantage. This is an issue that is complex and remains controversial due to conflicting research findings, many due to methodological differences between studies. Lovett [31] has highlighted central issues in the controversy over extended time accommodations and has reviewed some of the evidence to draw conclusions about the way extended time accommodations are currently provided. He argues for developing tests using principles of universal design so that all students can take the tests in the same format.

Sireci, Scarpati and Li [32] have provided a comprehensive review of studies that attempted to address the issue of test accommodations for students with disabilities and how they impact both students identified with disabilities and those without disabilities who do not receive accommodations. Their review supported the contention that extended time helps students with disabilities. They also found that extra time tends to improve the performance of all students, not just those with disabilities. However, they noted that students with disabilities tend to experience more substantial gains from extended time than do their non-disabled counterparts. They suggested further research to consider whether time limits should be extended for all students on most tests.

The purpose of this study was not to try to resolve the complex issues of fairness in allowing or denying use of accommodations to students with various types of impairments. It is to test hypotheses about the impact of ADHD-related executive function impairments on reading comprehension of adolescent students with ADHD and regarding the potential benefits of extended time for these students when taking tests requiring reading comprehension of more complex texts.

Our study indicated that many, but not all of our participants with ADHD were unable to complete one or both sections of the NDRT within standard time allotments. This suggests that some diagnosed with ADHD do not need extended time on tests such as the NDRT, though many do need extended time to have a fair chance to show what they know and can do.

These findings suggest that clinicians screening individuals for ADHD should include in their evaluation specific inquiries about reading comprehension, speed of reading, memory for what has been read, and whether the individual can usually finish tests and exams within usual time constraints. In making such inquiries, it is important that the clinician ask about assigned reading as distinguished from reading of self-chosen materials in which the person has strong personal interest.

As mentioned in the introduction to this paper, the situational variability of executive function impairments associated with ADHD can result in very different levels of functioning for self-chosen vs. assigned reading tasks [2]. For patients who report significant difficulties in reading speed, recall and comprehension of what has been read, or difficulty in completing tests or exams within standard time allotments, a more comprehensive assessment of reading, working memory and processing speed is usually indicated.

Current regulations on most high-stakes testing require clinical diagnostic interviews and a battery of psychoeducational testing for persons requesting extended time for such exams based on diagnosis of ADHD or a specific learning disorder. Such an assessment seems appropriate to establish need for extended time, but this requirement is problematic for students who cannot afford the high costs of such testing which often is not covered by insurance. The requirement is also problematic for those who do not have access to psychological evaluators who have the appropriate expertise.

\subsection{Limitations of the Study}

Findings from this study should be understood in light of limitations of the study.

First, this sample is characterized by students who tended to have high average verbal comprehension abilities. Applicability to students with different levels of verbal comprehension may be limited and remains to be tested.

The Nelson-Denny Reading Test has been criticized for having a low ceiling which does not provide adequate challenge for students of stronger ability who may be required in their schooling to read and take tests involving much more complex text than is provided by the 
Nelson-Denny. Also, one study by Coleman, et al. [33] found that a sample of students with and without disabilities were able to obtain scores well above chance on this test without having read the passages on which the comprehension questions are based.

However, despite its limitations, the NDRT is one of the few reading tests currently available that provides passages of text that extend for several paragraphs. Most other reading comprehension tests currently available attempt to test reading comprehension, even for adolescent and adult students, using very short passages or, in some cases, single sentences that bear little relationship to the longer, more complex readings required even of junior high and high school students, not to mention students in college or university programs. The NDRT also offers two normed versions and provides a useful measure for both a standard and extended time conditions.

Clearly more adequate normed measures are needed, but at this time the NDRT, despite its very real limitations, provides a more challenging normed measure of reading comprehension for adolescents and adults than do most other measures of reading comprehension available at this time.

\section{CONCLUSIONS}

Given the findings of this study, it would seem advisable for clinicians assessing individuals with ADHD to inquire directly about whether they are able to complete tests involving reading comprehension within the time usually allowed. If the student reports frequent inability to complete such tests, the student should be referred for a full psychoeducational evaluation, including the NDRT or a comparable measure, to establish whether accommodations including extended time for tests and examination are appropriate and should be provided.

\section{REFERENCES}

[1] Pennington, B.F. (1991) Diagnosing learning disorders: A neurological framework. Guilford Press, New York.

[2] Brown, T.E. (2005) Attention deficit disorder: The unfocused mind in children and adults. Yale University Press, New Haven.

[3] Brown, T.E. (2009) Developmental complexities of attentional disorders. In T.E. Brown (Ed.), ADHD Comorbidities: Handbook for ADHD complications in children and adults. American Psychiatric Publishing, Inc., Washington, D.C., 3-22.

[4] Anmarkrud, O. and Braten, I. (2009) Motivation for reading comprehension. Learning and Individual Differences, 19, 252-256. doi:10.1016/j.lindif.2008.09.002

[5] Willcutt, E.G., Pennington, B.F., et al. (2005) Neuropsychological analyses of comorbidity between reading disability and attention deficit hyperactivity disorder: In search of the common deficit. Developmental Neuropsy- chology, 27, 35-78.

doi:10.1207/s15326942dn2701 3

[6] Shanahan, M.A., Pennington, B.F., et al. (2006) Processing speed deficits in attention deficit/hyperactivity disorder and reading disability. Journal of Abnormal Child Psychology, 34, 585-602. doi:10.1007/s10802-006-9037-8 2

[7] Laasonen, M., Leppamaki, S., et al. (2009) Adult dyslexia and attention deficit disorder in Finland-project DyAdd. Journal of Learning Disabilities, 42, 511-527. doi:10.1177/0022219409345013

[8] McGrath, L.M., Pennington, B.F., et al. (2011) A multiple deficit model of reading disability and attention-deficit-hyperactivity disorder: Searching for shared cognitive deficits. Journal of Child Psychology and Psychiatry, 52, 547-557

doi:10.1111/j.1469-7610.2010.02346.x

[9] Arnell, K.M., Joanisse, M.F., et al. (2009) Decomposing the relation between rapid automatized naming (RAN) and reading ability. Canadian Journal of Experimental Psychology, 63, 173-184. doi:10.1037/a0015721

[10] Swanson, H.L., Zheng, X., et al. (2009) Working memory, short-term memory, and reading disabilities. Journal of Learning Disabilities, 42, 260-287. doi:10.1177/0022219409331958

[11] Sesma, H.W., Mahone, E.M., et al. (2009) The contribution of executive skills to reading comprehension. Child Neuropsychology, 15, 232-246. doi:10.1080/09297040802220029

[12] Locascio, G., Mahone, M., Eason, S. and Cutting, L. (2010) Executive dysfunction among children with reading comprehension deficits. Journal of Learning Disabilities, 43, 441-454. doi: $10.1177 / 0022219409355476$

[13] Samuelsson, S., Lundberg, I., et al. (2004) ADHD and reading disability in male adults: Is there a connection? Journal of Learning Disabilities, 37, 155-168. doi:10.1177/00222194040370020601

[14] Jacobson, L.A., Matthew, R., et al. (2011) Working memory influences processing speed and reading fluency in ADHD. Child Neuropsychology, 17, 209-224. doi:10.1080/09297049.2010.532204

[15] Bental, B. and Tirosh, E. (2007) The relationship between attention, executive functions and reading domain abilities in attention deficit hyperactivity disorder: A comparative study. Journal of Child Psychology and Psychiatry, 48, 455-463. doi:10.1111/j.1469-7610.2006.01710.x

[16] Leong, C.K., Tse, S.K., et al. (2008) Text comprehension in chinese children: Relative contribution of verbal working memory, pseudoword reading, rapid automatized naming, and onset-rime phonological segmentation. Journal of Educational Psychology, 1, 135-149. doi:10.1037/0022-0663.100.1.135

[17] Willcut, E., Pennington, B., Boada, R., Ogline, J., Tunick, R. and Chhabildas, N. (2001) A comparison of the cognitive deficits in reading disability and attention-deficit/ hyperactivity disorder. Journal of Abnormal Psychology, 110, 157-172. doi:10.1037//0021-843X.110.1.157

[18] Cain, C. and Oakhill, J. (2007) Reading comprehension difficulties: Correlates, causes and consequences. In chil- 
dren's comprehension problems in oral and written language. Guilford Press, New York, 41-75.

[19] Shaywitz, S.E. and Shaywitz, B.A. (2008) Paying Attention to Reading: The neurobiology of reading and dyslexia. Development and Psychopathology, 20, 1329-1349. doi:10.1017/S0954579408000631

[20] Walczyk, J.J., Wei, M., et al. (2007) Development of the interplay between automatic processes and cognitive resources in reading. Journal of Educational Psychology, 99, 867-887. doi:10.1037/0022-0663.99.4.867

[21] Mayes, S.D. and Calhoun, S.L. (2007) Wechsler intelligence scale for children-third and -fourth edition predictors of academic achievement in children with attention-deficit/hyperactivity disorder. School PsychologyQuarterly, 22, 234-249. doi:10.1037/1045-3830.22.2.234

[22] Brown, T.E. (1996) Brown attention deficit disorder scales for adolescents and adults. TX. The Psychological Corporation, San Antonio.

[23] Brown, T.E. (2001) Brown Attention Deficit Disorder Scales for Children and Adolescents. The Psychological Corporation, San Antonio.

[24] Wechsler, D. (2003) Wechsler intelligence scale for children-fourth edition. TX. The Psychological Corporation, San Antonio.

[25] Wechsler, D. (1997) Wechsler adult intelligence scalethird edition administration and scoring manual. The Psychological Corporation, San Antonio.

[26] Wechsler, D. (2002) Wechsler individual achievement test-second edition. TX. Psychological Corporation, San Antonio.

[27] Woodcock, R.W., McGrew, K.S., et al. (2001) Wood- cock-johnson-third edition tests of achievement. IL. Riverside Publishing, Itasca.

[28] Mayes, S.D., Calhoun, S.L., et al. (2009) ADHD subtypes and co-occurring anxiety, depression, and oppositional defiant disorder: Differences in gordon diagnostic system and wechsler working memory and processing speed index scores. Journal of Attention Disorders, 12, 540-549. doi:10.1177/1087054708320402

[29] Brown, J.I., Fishco, V.V. and Hanna, G. (1993) Nelson-denny reading test. Riverside Publishing Co., Chicago.

[30] Ofiesh, N., Mather, N. and Russell, A. (2005) Using speeded cognitive, reading, and academic measures to determine the need for extended test time among university students with learning disabilities. Journal of Psychoeducational Assessment, 23, 35-52. doi: $10.1177 / 073428290502300103$

[31] Lovett, B.J. (2010) Extended time testing accommodations for students with disabilities: Answers to five fundamental questions. Review of Educational Research, 80, 611-638. doi:10.3102/0034654310364063

[32] Sireci, S.G., Scarpati, S.E. and Li, S. (2005) Test accommodations for students with disabilities: An analysis of the interaction hypothesis. Review of Educational Research, 75, 457-490. doi: $10.3102 / 00346543075004457$

[33] Coleman, C., Lindstrom, J., Nelson, J., Lindstrom, W. and Gregg, K. (2010) Passageless comprehension on the nelson-denny reading test: Well above chance for university students. Journal of Learning Disabilities, 43, 244249. doi:10.1177/0022219409345017 